\title{
Coronavirus-2019 (Covid 19): A Review of History, Epidemology, Structure and Life Cycle
}

\author{
Israa Abdul Ameer Al-Kraety ${ }^{1}$, Sddiq Ghani Al-Muhanna², Aaya Hamid Al-Hakeem \\ ${ }^{1}$ Lect., ${ }^{2}$ Lect., ${ }^{3}$ Assist. Lect., Department of Medical Laboratory Techniques, \\ Faculty of Medical and Health Techniques, University of Alkafeel, Najaf, Iraq
}

\begin{abstract}
COVID-19, a new, rapidly spreading coronavirus strain, has reached more than 150 countries and is gaining worldwide attention. The shortage of successful SARS-CoV-2 medicines or vaccines has exacerbated the situation even further. Therefore, research is urgently required to establish effective therapeutics and affordable diagnosis For COVID-19. It is the responsibility of the scientific research community in this time of health crisis to provide an alternative, reliable, and accessible method for vaccinating human bodies against COVID-19 viral infections, based on focused experimental approaches. Corona virus (CoV) is an RNA virus for the forward,forming stick-shaped spikes on its surface. This is an undesirable, small RNA genome, with an infinite mode of replication. The corona virus causes numerous diseases in mammals, birds, pigs, and chickens. It causes upper respiratory tract infections, which can lead to death from respiratory diseases. Within this article the author briefly explains this abrupt occurrence Extreme lung disease, extremely pathogenic and newly discovered respiratory syndrome (MERS-CoV) in corona virus of the Middle East. It is a research paper on the detection of CoVID-19 infections and their dissemination across the world.
\end{abstract}

Keywords: Covid-19, Structure of coronavirus and Life cycle.

\section{Introduction}

Hospitals declared on 31 December 2019 in Wuhan, Hubei, China, a cluster of cases of unknown cause of pneumonia attracting great national and worldwide attention $^{(1)}$. Coronavirus $(\mathrm{CoV})$ is a big group of positive Nidoviral RNA single-stranded viruses. There is also a list of the Arteriviridae, Coronaviridae, and Roniviridae. The Coronaviridae family is classified in the Coronavirinae and Torovirinae subfamilies. Coronavirinae even below the alpha, beta, gamma, and delta COV level ${ }^{(2)}$. Such forms of viruses have been

\footnotetext{
Corresponding Author: Sddiq Ghani Al-Muhanna

Lect., Department of Medical Laboratory Techniques, Faculty of Medical and Health Techniques, University of Alkafeel, Najaf, Iraq e-mail: sddiq.g@gmail.com
}

named for the phylogenetic clustering. The viral RNA genome varies in length from 26 to 32 kilobases. They can be separated from various species of animals. These involve birds, goats, and mammals including camels, bats, and masks Civets of leaves, mice, pigs, cats.. The widely distributed distribution and infectiveness of COV makes it a major pathogen. The moderate clinical signs are associated with human pathogenic subtypes of $\mathrm{CoV}$. Yet extreme coronavirus-related acute respiratory syndrome (SARS-CoV) and the Middle East The two notable exceptions are respiratory coronavirus syndrome (MERS-CoV). In Saudi Arabia MERS-CoV was first observed in 2012. It had 2,494 confirmed cases leading to 858 deaths. In 2002, a Beta-COV subtype spread rapidly in Guangdong, China. In 37 countries this epidemic resulted in 8,000 infections and 774 deaths $)^{3(\cdot}$. In Wuhan, China, in the form of unknown etiology pneumonia, the outbreak in 2020 presented. The culprit was identified as a new COV strain by deep-sequencing experiments and laboratory tests. It was originally known as the 2019$\mathrm{nCoV}$ virus. It was listed as the SARS-CoV-2 virus by the International Committee on Virus taxonomy. The 
World Health Organization (WHO) named the disease on 11 February 2020 as the coronavirus disease-2019 (COVID-19), caused by this novel virus. The repeated appearance and $\mathrm{CoV}$ outbreaks suggest a threat to public health. This demonstrates the possibility of moving newly evolving CoVs from animal to human, and human to human. The ongoing ecological and climatic changes make these infections more likely to occur in the future. ${ }^{(4)}$

History of Coronavirus: (1960), it reported the very first corona virus outbreak with cold symptoms. According to the Canadian study of 2001, approximately 500 patients were registered as pneumonia-like conditions. In 2003, numerous studies have been published with proof of the expansion of the corona to various states such as Hong Kong, the United States, Thailand,Singapore, and China. In 2003, several cases of a severe acute respiratory syndrome caused by the corona were reported, and in March to June 2003, their death rate was around 1000 patients. In 2012 Saudi Arabian reported multiple infectious victims and deaths ${ }^{(5)}$. COVID-19 was first observed and reported from the pneumonia monopoly belonging to China, Wuhan. Much of the sudden outbreak occurred..In Hong Kong, China,Singapore and other countries in North America andSoutheast Asia. In December 2019, this epidemic broke out again in the city of Wuhan (China). $\mathrm{NCoV}-19$ was approximately distributed worldwide to more than a hundred counties. Of all nations, 80,824 cases $(54.02 \%)$ confirmed by nCoVD-19 are reported and their incidence continually increased ${ }^{6}$.

Epidemiology of Covid -19: The coronaviruses are the single-stranded RNA viruses common to humans, other animals, and birds that cause enteric, liver, breathing, and neurologic problems.Six coronavirus species are known to cause human disease. Four viruses such as hCoV-229E, OC43, NL63, and HKU1 are prevailing and typically cause mild illnesses ${ }^{[7]}$. The two new lethal diseases (coronaviruses), the 2002 severe acute respiratory coronavirus syndrome (SARS-CoV), and the 2012 Middle East Respiratory Coronavirus Syndrome (MERS-CoV), are widespread at different locations. The occurrence of new coronaviruses and the broad spread of coronavirus, genetic variation, regular genome recombination, and human and animal behavior are also correlated with the development of new coronaviruses in humans. As $)^{8(}$ the $2019-n C o V$ genome produced is therefore phylogeneously closest to other SARS-CoV identified beta-coronavirus, which according to other studies suggests are $75-80 \%$ identical to the SARS-CoV and $40 \%$ equivalent to MERSCoV. This could be propagated by increasing SARS-CoV and MERS-CoV in the coronaviridae family. Indeed, in primary airway cells of the 2019-nCoV epithelial cell, as opposed to SARS-CoV or MERS-CoV, grow better than standard tissue-culture cells. It seems that 2019-nCoV has the same hACE2 (human angiotensin-converting enzyme 2) cellular receptor as SARS-CoV ${ }^{8}($.

Structure genome coronavirus: The genomic structure of the coronavirus is a member of the Nidoviral, the Coronaviridae, and Coronavirinae subfamily. This subfamily is also known as coronaviruses on the basis of the phylogenetic cluster of alpha, beta, gamma, and delta. These viruses are found in RNA enveloped by 26-32 kilobases. The genome has a $5^{\prime}$ cap and a $3^{\prime}$ poly(A) tail to help serve as mRNA in the translation of polyprotein replicas $^{(2)}$. In addition to an untraduced region and a leading sequence, the $5^{\prime \prime}$ coronavirus end comprises several loop structures that contribute to transcription and replication of the RNA. There are also transcriptional regulatory sequences at the beginning of each structural gene which supports their expression. Even the 3'UTR segment includes RNA structures that help replicate and synthesize viruses. Coronavirus accessory proteins are not needed for replication; however, it was demonstrated that some of the protein in viral pathogenesis played significant roles $^{(9)}$. Coronavirus virions, as confirmed by cryo-electrons microscopic and tomographical techniques, have spherical form and a diameter of about $125 \mathrm{~nm}$. The form of the club is like projections on the floor. The nucleocapsid inside the virus is symmetrically symmetric, which is characterizing the negative nature of RNA virus disease ${ }^{(2)}$. Virions are four main structures, namely the pike, membrane, shell, and nucleocapsid, in the $3^{\prime}$ end of the viral genome. The $\beta$-coronavirus group also contained a fifth structural protein, hemagglutinin esterase. It binds sialic acids to a glycoprotein's surface and is involved in acetylesterase. These activities improve protein-mediated cell entry and distribution in $\mathrm{S}$. 


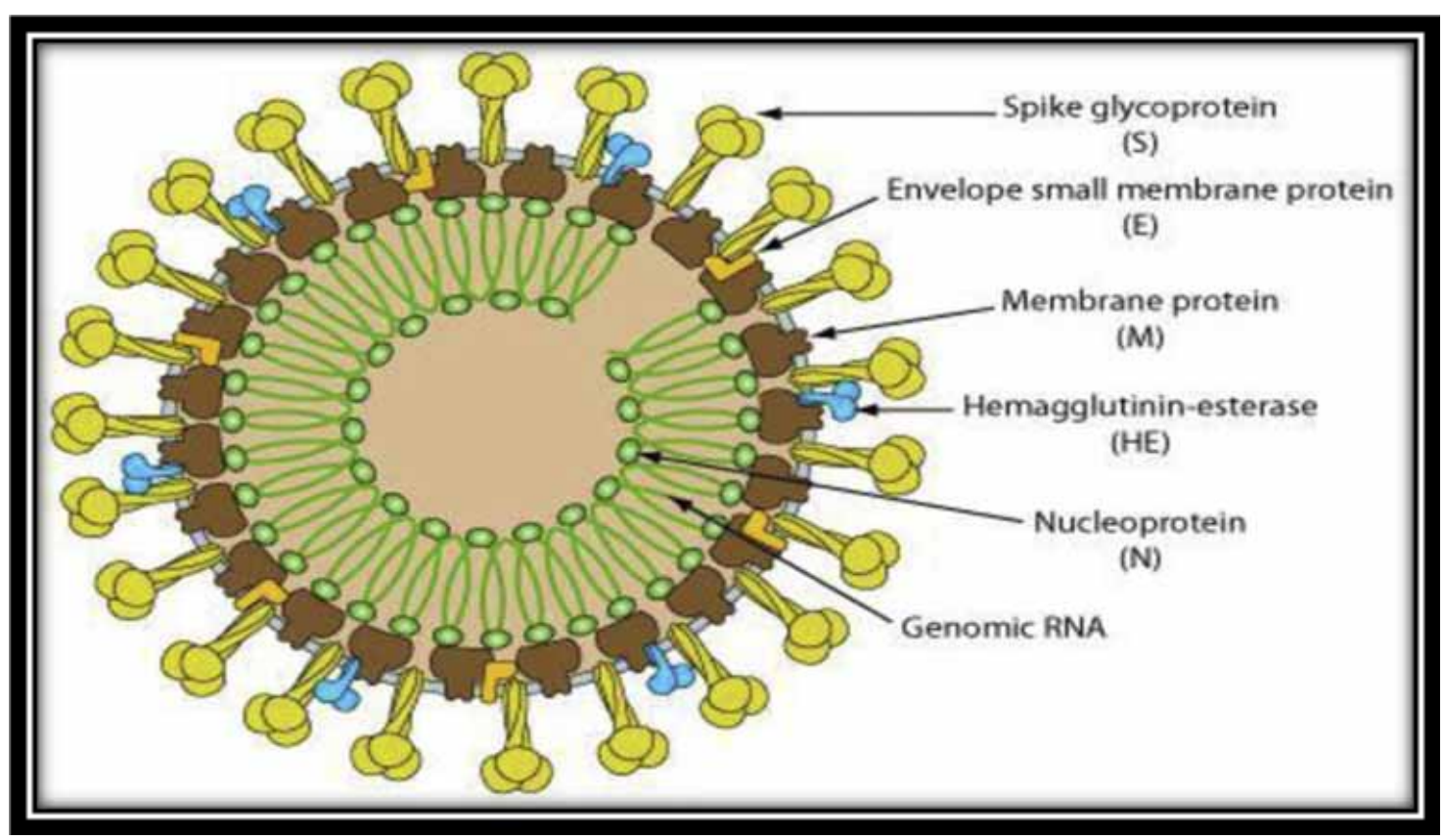

Figure 1: Structure of Coronvirus. From Biowiki (http://ruleof6ix. fieldofscience.com/2012/09/a-newcoronavirus-should-youcare.html).

Coronavirus Life Cycle: The four stages of a coronavirus life cycle are: (1) entry and attachment (2) protein expression replicase (3) replication and transcription (4) assembly and release. The association of the S-proteins with their receptors marks the virion's initial attachment to the host cell. The S-Protein and the receiver mixture is the primary determinant of a coronavirus that also affects the hosts and controls the virus' tissue tropism. In most coronaviruses, peptidase serves as the cell receptor. The receptor reaches the cytosol in the host cell once the virus has been released ${ }^{4}$ (. This action is facilitated by the acid-dependent S-protein proteolytic cleavage called cathepsin or other associated proteases accompanied by cellular and viral membrane fusion. Two cleavages are formed in the S2 portion of the protein; one is necessary in order to distinguish the receptor binding and the S-protein fusion domain and another is needed for exposure to peptides.Most of the fusion however occurs in acidified endosomes, some coronaviruses fusing on the plasma membrane region. Virion entry and attachment is accompanied by the expression of replicase protein, in which the replicase gene is converted into the virion genomic RNA. The replicase gene encodes two ORFS; repla and rep1b, which in turn are expressed in two co-terminal polyproteins, pp 1a and pplab. The virus uses a slippery sequence to express all the polyproteins (5'-UUUAAAC-3') and a pseudoknot of RNA that triggers ribosomal frameshifting from the rep1a read frame to the rep1b ORF) ${ }^{10(.}$ The ppla and pplab polyproteins comprise the 1-11 and 1-16 nsps, respectively. In pplab, after extension of ppla into pp1b, nsp11 from pp1a becomes nsp12. The nsps are assembled in the replicate-transcriptase complex (RTC), the setting in which the synthesis of RNAs is conceived and the replication and transcription of subgenomic RNAs are primarily responsible for these. Create and translate the genome and subgenomic RNAs after virus RNA-synthesis. Subgenomic RNAs reflect the accessory and structural genes as mRNAs occurring downstream of replicas of polyproteins. Both genomic, as well as subgenomic RNAs, are produced by negativestrand intermediates. Finally, coronaviruses also have remarkable capacity to recombine with homologous and non-homologous recombinations ${ }^{(11)}$. After replication and subgenomic synthesis of the RNA, S, E, and M viral structure proteins are encoded and incorporated into the endoplasmic reticulum (ER). Such proteins can pass through the secretory pathway into the endoplasmic reticulum-Golgi intermediate compartment (ERGIC). The N-protein bud encapsulates viral genomes into ERGIC membranes containing the viral structural proteins that form mature virions These virions are transported to the cell surface and released via the process of exocytosis $^{(12)}$. 


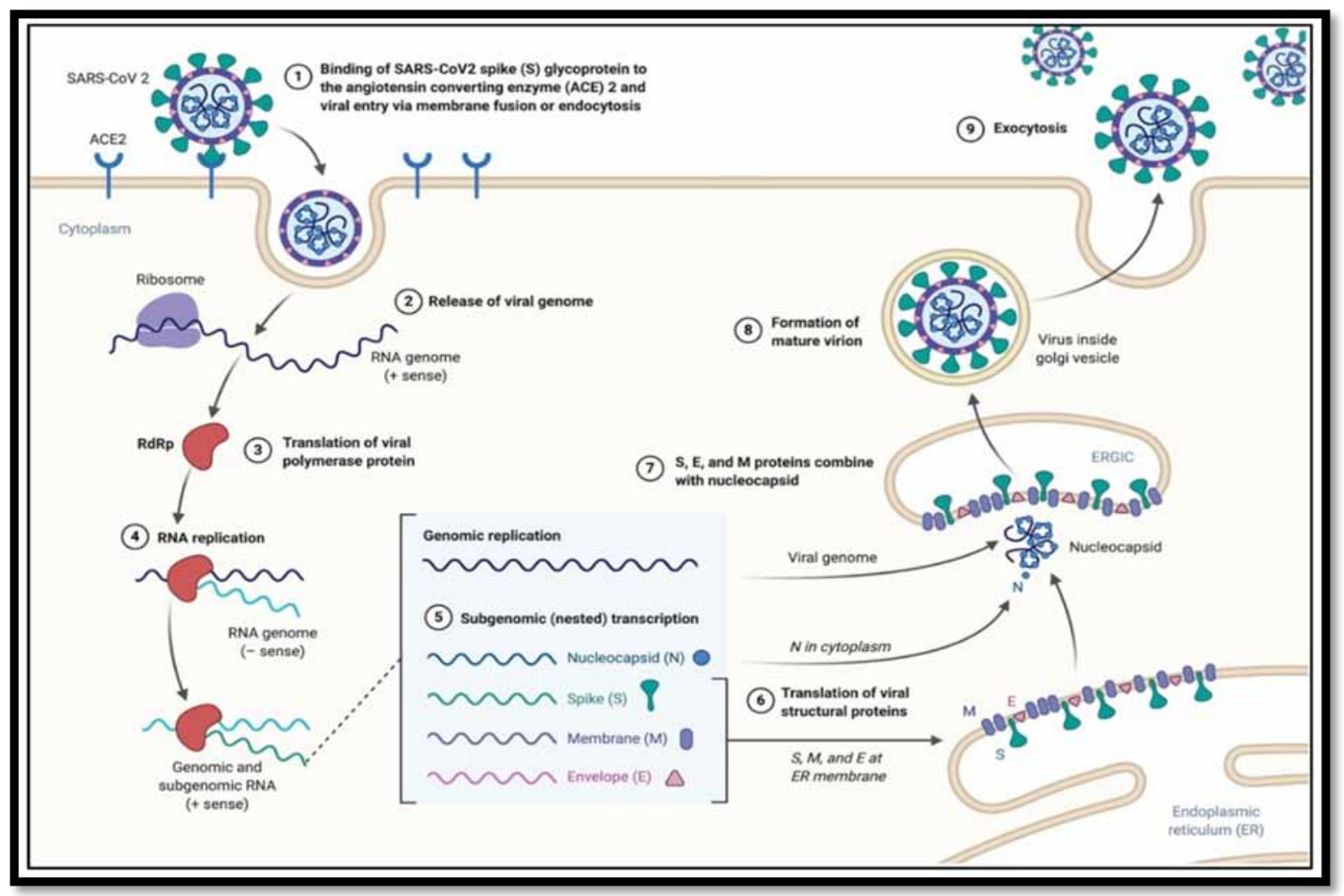

Figure 2: The SARS-CoV-2 life cycle in host cells (figure created with biorender.com).

\section{Conclusions}

The pandemic in COVID-19 is a significant challenge to healthcare systems worldwide. The international community was alerted to future worstcase situations by the number of injuries and deaths. We have no clear information ex Strategy to treat COVID-19 patients despite our bits of knowledge of the SARS-CoV-2 infectious cycles. Different potential drugs with SARS-CoV-2 anti-viral activity are studied on the basis of recent research results and guidance. Ideally, an appropriate vaccine and best practices will be established to diagnose and treat symptomatic cases.

Ethical Clearance: The Research Ethical Committee at scientific research by ethical approval of both MOH and MOHSER in Iraq.

\section{Conflict of Interest: None}

Funding: Self-funding

\section{Reference}

1. Wang C, Horby PW, Hayden FG, Gao GF.A novel coronavirus outbreak of global health concern. Lancet. 2020)) Jan 14.
2. Fehr AR, Perlman S. Coronaviruses: an overview of their replication and pathogenesis.(2015).

3. Lu R, Zhao X, Li J, et al. Genomic characterisation and epidemiology of 2019 novel coronavirus: implications for virus origins and receptor binding. (2020).

4. Chen Y, Liu Q, Guo D. Emerging coronaviruses: genome structure, replication, and pathogenesis. (2020).

5. Peiris, J. S., K. Y. Yuen, A. D. Osterhaus and K. Stohr. The severe acute respiratory syndrome. The New England journal of medicine. (2003).

6. Bradburne, A. F., M. L. Bynoe and D. A. J. Tyrell. Effects of a "new" human respiratory virus in volunteers. (2006).

7. Su S, Wong G, Shi W et al. Epidemiology, genetic recombination, and pathogenesis of coronaviruses. Trends Microbiol(2016). 24:490-502.

8. Zhu N, Zhang DY, Wang WL et al.A novel coronavirus from patients with pneumonia in China, 2019. N Engl J Med.(2020).

9. Zhao L, Jha BK, Wu A, et al. Antagonism of the interferon-induced OAS-RNase L pathway by 

murine coronavirus ns 2 protein is required for virus replication and liver pathology. Cell host microbe (2012); 11: 607-616.

10. Araki K, Gangappa S, Dillehay DL, Rouse BT, et al. Pathogenic virus-specific $\mathrm{T}$ cells cause disease during treatment with the calcineurin inhibitor FK506: implications for transplantation.(2010).
11. Keck JG, Makino S, Soe LH, et al.RNA recombination of coronavirus. Adv Exp Med Bio (1987). 218: 99-107.

12. De Haan CA, Rottier PJ. Molecular interactions in the assembly of coronaviruses. Adv Virus Res (2005); 64: 165-230. 